\title{
THE HRUSHOVSKI PROPERTY FOR HYPERTOURNAMENTS AND PROFINITE TOPOLOGIES
}

\author{
JINGYIN HUANG, MICHAEL PAWLIUK, MARCIN SABOK, AND DANIEL WISE
}

\begin{abstract}
We study the problem of extending partial isomorphisms for hypertournaments, which are relational structures generalizing tournaments. This is a generalized version of an old question of Herwig and Lascar. We show that the generalized problem has a negative answer, and we provide a positive answer in a special case. As a corollary, we show that the extension property holds for tournaments in case the partial isomorphisms have pairwise disjoint ranges and pairwise disjoint domains.
\end{abstract}

\section{INTRODUCTION}

In [12] Hrushovski showed the following property for finite graphs: whenever $G$ is a finite graph and $\varphi_{1}, \ldots, \varphi_{n}$ are partial isomorphisms of $G$, there exists a finite graph $G^{\prime}$ containing $G$ as an induced subgraph such that $\varphi_{1}, \ldots, \varphi_{n}$ all extend to automorphisms of $G^{\prime}$. This property appears in the literature under various names (e.g. as the EPPA for Extending Property for Partial Automorphisms or simply as the Hrushovski property) and we say that a class $C$ of structures has the Hrushovski property if for any structure $M$ in $C$ and a finite collection $\varphi_{1}, \ldots, \varphi_{n}$ of partial isomorphisms of $M$ there exists a structure $M^{\prime}$ in $C$ which contains $M$ as a substructure and such that all $\varphi_{i}$ extend to automorphisms of $M^{\prime}$. A general criterion sufficient for the Hrushovski property was given by Herwig and Lascar [10] for structures in finite relational languages and also by Hodkinson and Otto in [11. Recently, both these theorems were generalized by Siniora and Solecki in [4]. The Hrushovski property was also studied and extended to other classes of homogeneous structures, for instance by Solecki to the class of finite metric spaces [26] (for other proofs see [28, 22, 24, 25, 13]) or by Evans, Hubička, Konečný and Nešetřil [6, 5]. For a detailed discussion of this and related problems the reader is advised to consult a recent survey of Nguyen Van Thé [21] on the topic or the ICM survey article of Lascar [18].

This research was partially supported by the FRQNT (Fonds de recherche du Québec) grant Nouveaux chercheurs 2018-NC-205427 and by the NCN (Polish National Science Centre) through the grant Harmonia no. 2015/18/M/ST1/00050. 
The Hrushovski property for a Fraïssé class of finite structures is useful for the study of automorphism groups of the corresponding Fraïssé limits. A topological group $G$ has ample generics if for each $n$ there exists a dense $G_{\delta}$ orbit in the diagonal action of $G$ on $G^{n}$ (i.e. the action $\left.g \cdot\left(g_{1}, \ldots, g_{n}\right)=\left(g g_{1}, \ldots, g g_{n}\right)\right)$. Ample generics have strong consequences, such as the automatic continuity property of the group. Kechris and Rosendal [15] gave a general criterion sufficient for ample generics in an automorphism group of a Fraïssé structure that involves the Hrushovski property for the corresponding Fraïssé class. In particular, they used the original Hrushovski property for finite graphs in showing that the automorphism group of the random graph has ample generics, and consequently the automatic continuity property.

Fraïssé classes of graphs have been classified by Lachlan and Woodrow [17] and for directed graphs a complete classification has been given by Cherlin [2]. The Hrushovski property for many classes of directed graphs has been studied in the literature and proved or disproved for many classes. In fact, the Hrushovski property implies amenability of the automorphism group of the automorphism group of the appropriate Fraïssé structure. The latter does not hold for the linear tournament, the generic p.o., some weak local orders (see e.g [20, page 4] for details).

The question for the class of tournaments is well-known and open and appears in the Herwig and Lascar paper [10].

Question 1.1. (Herwig and Lascar [10]) Does the class of finite tournaments have the Hrushovski property?

This question is related with the problem whether the automorphism group of the random tournament has ample generics. In fact, as proved recently by Siniora [3], the two questions are equivalent. It is worth noting that the automorphism group of the random tournament has a comeager conjugacy class (see e.g. [19]).

This paper we show that the Hrushovski property does hold for tournaments in a special case when we make an extra assumption on the partial automorphisms. In fact, this works in a more general setting of hypertournametns (for definitions see Section 7).

Theorem 1.2. Suppose $L$ is a nontrivial set of prime numbers and $M$ is an L-hypertournament. If $\varphi_{1}, \ldots, \varphi_{n}$ are partial automorphisms of $M$ with pairwise disjoint domains and pairwise disjoint ranges, then there is an $L$ hypertournament $M^{\prime}$ extending $M$ such that all $\varphi_{i}$ extend to automorphisms of $M^{\prime}$.

While the above result does cover the case of tournaments (when $L=$ $\{2\}$ ), we show that the assumption on domains and ranges of the partial automorphisms cannot be dropped completely.

Theorem 1.3. It is not true that the Hrushovski property holds for all classes of L-hypertournaments. 
Herwig and Lascar [10] connected the question about tournaments with a problem concerning profinite topologies on the free group. A group $G$ is called residually finite if for every $g \in G$ with $g \neq e$ there exists a finite-index subgroup $H$ of $G$ such that $g \notin H$. It is well known that the free groups are residually finite. The profinite topology on a residually finite group is the one with the basis neighborhood of the identity consisiting of finite-index subgroups. A subgroup $H$ of a residually finite group is separable if it is closed in the profinite topology and a group $G$ is $L E R F$ if all its finitely generated subgroups are separable. Free groups are LERF, by a result of Hall [8].

Herwig and Lascar [10] found a proof of Hrushovski's theorem using the fact that free groups are LERF and the Ribes-Zalesskii theorem saying that products of f.g. subgroups are closed in the profinite topology on the free group. In a similar spirit, they showed that Question 1.1 is equivalent to the following Question 1.4. Here, we say that a subgroup $H$ of $G$ is closed under square roots if whenever $g^{2} \in H$, then $g \in H$ for any $g \in G$ and the odd adic topology is a refinement of the profinite topology where we take only finite index normal subgroups of odd index as the basic neighborhoods of the identity.

Question 1.4. (Herwig and Lascar [10]) Is it true that for every finitely generated subgroup $H$ of the free group $F_{n}$ the following are equivalent:

(i) $H$ is closed in the odd-adic topology on $F_{n}$,

(ii) $H$ is closed under square roots?

The implication from (i) to (ii) above is true and not very difficult, so the actual part of Conjecture 1.4 that is equivalent to Conjecture 1.1 is the implication from (ii) to (i).

The proof of Theorem 1.2 is based on showing that the answer to the above question is true for cyclic subgroups of $F_{n}$ even in a more general context (for definitions see Sections 3 and 7 ).

Theorem 1.5. Let $C<F_{n}$ be a cyclic subgroup and $L$ be a nontrivial set of prime numbers. If $C$ is closed under l-roots for any $l \in L$, then $C$ is closed in the topology generated by the collection of pro-p topologies with $p$ ranging over $L^{\perp}$.

On the other hand, the negative result in Theorem 1.3 is based on the following result (for definitions see Section 2).

Theorem 1.6. There exists a malnormal subgroup of $F_{2}$ which is not closed in any pro-p topology.

\section{Preliminaries}

A graph is a 1-dimensional cell $(\mathrm{CW})$ complex, in which vertices are 0cells (points) and edges are the 1-cells (intervals) glued to the 0-skeleton by their end points. Loops and multiple edges allowed. A morphism of 
graphs is a cellular map that sends each open edge (1-cell without endpoints) homeomorphically onto an open edge. A graph morphism $f: X \rightarrow X^{\prime}$ is an immersion if it is locally injective (i.e. each point has a neighborhood on which $f$ is injective). When $X$ and $X^{\prime}$ are connected, an immersion between them induces injective maps between their fundamental groups.

Note that if we label the circles in the wedge of $n$ many circles with letters $a_{1}, \ldots, a_{n}$, then we can pull back this labelling to a labelling of the graph immersed to the wedge of $n$ circles. Here we use the convention that if an edge is labelled with $a_{i}$, then the reverse edge is labelled with $a_{i}^{-1}$. By an immersed graph with $n$ letters we mean a directed graph with edges labelled with one of the $n$ letters, say $a_{1}, \ldots, a_{n}$ such that for each vertex and $i \leq n$ there is at most one incoming edge labelled with $a_{i}$ and at most one outgoing edge labelled with $a_{i}$. Note that immersed graphs with $n$ letters on a vertex set $X$ correspond to the sets of $n$-many partial bijections of $X$. Note also that an immersed graph with $n$ letters is a cover of the bouquet of $n$ circles if and only if the partial bijections are total. Given an immersed graph on $X$ with $n$ letters and $x_{0} \in X$, the fundamental group $\pi_{1}\left(X, x_{0}\right)$ is the subgroup of $F_{n}=\left\langle a_{1}, \ldots, a_{n}\right\rangle$ consisting of those words on $a_{1}, \ldots, a_{n}$ which form loops at $x_{0}$.

The following standard theorem (which follows from the work of Stallings) shows that any finitely generated subgroup of $F_{n}$ is of the above form.

Theorem 2.1. Write $X_{n}$ for the wedge of $n$-circles with its based vertex $x_{n}$. For any finitely generated subgroup $H \leq F_{n}$, there is a based finite graph $(X, x)$ and an immersion

$$
f:(X, x) \rightarrow\left(X_{n}, x_{n}\right)
$$

such that $\pi_{1}(X, x) \cong H$ and $f_{*}: \pi_{1}(X, x) \rightarrow \pi_{1}\left(X^{\prime}, x^{\prime}\right)$ is exactly the inclusion $H \leq F_{n}$.

Given a graph $X$ and a ring $R$, by its first chain group $C_{1}(X, R)$ we mean the cellular chain group, where each edge gives a generator in the cellular chain group. We will always view the first homology group $H_{1}(X, R)$ as a subgroup of the first chain group $C_{1}(X, R)$ (for details see e.g. 9, Chapter 2.2.]).

Given a goup $G$ and a space $X$, we say that a covering space $\hat{X} \rightarrow X$ is a $G$-cover if it is a regular cover and the deck transformation group is isomorphic to $G$.

We will also need the following Nielsen-Schreier formula.

Theorem 2.2 (14]). Let $H$ be a subgroup of index $k$ inside a free group on $n$ generators. Then $H$ is a free group of rank $1+k(n-1)$.

Definition 2.3. A subgroup $H$ of $G$ is malnormal if for any $g \in G \backslash H$, we have $H \cap g H g^{-1}=\{1\}$.

Definition 2.4. Given a positive integer $l$, we say that a subgroup $H$ of $G$ is closed under l-roots if for every $g \in G$ whenever $g^{l} \in H$, then $g \in H$. 
If $l=2$, then we refer to the above by saying that $G$ is closed under square roots.

Claim 2.5. If $G$ is torsion-free and $H \leq G$ is malnormal, then $H$ is closed under l-roots for all $l$.

Proof. Suppose $g^{l} \in H$ but $g \notin H$. Then $H \cap g H g^{-1}=\{1\}$ by malnormality. However, $H \cap g H g^{-1}=\{1\}$ contains the infinite cyclic group generated by $g^{l}$, which is a contradiction.

\section{Profinite topologies}

We consider several profinite topologies on the free group.

Definition 3.1. Given a set $P$ of prime numbers consider the topology on the free group generated by cosets of those normal subgroups whose index is finite and divisible only by prime numbers in $P$. We refer to this topology as to the pro- $P$ topology.

Note that the neighborhoods of the identity in the pro- $P$ topology are those normal subgroups $H$ of finite index such that all orders of elements of $F_{n} / H$ are divisible only by numbers in $P$.

In the case $P$ consists of a single prime number $p$, we get the pro- $p$ topology on the free group. Another special case when $P$ consists of all odd primes was considered by Herwig and Lascar [10] who considered the above topology under the name of the odd-adic topology. For more on the pro- $P$ topologies, the reader can consult [23].

Recall that a group $G$ is residually $p$ if the trivial subgroup is closed in the pro- $p$ topology of $G$. The free group $F_{n}$ is residually $p$ for any prime $p$ (for a short proof see e.g. [16, Lemma 2.23]).

Definition 3.2. Given a set $L$ of positive integers write

$$
L^{\perp}=\{p \text { prime } \mid(p, l)=1 \text { for any } l \in L\} .
$$

We say that $L$ is a nontrivial set of positive integers if $L^{\perp} \neq \emptyset$.

Claim 3.3. If $H \leq F_{n}$ is closed in the pro- $L^{\perp}$ topology, then $H$ is closed under l-roots for all $l \in L$.

Proof. Indeed, if $g^{l} \in H$ but $g \notin H$, then $g$ cannot be separated from $H$ in a finite quotient of rank relatively prime with $l$ because for an element $\bar{g}$ of such a group the subgroups generated by $\bar{g}^{l}$ and $\bar{g}$ are the same.

\section{Fiber PRODUCTS OF GRAPHS}

Definition 4.1. Let $A \rightarrow X$ and $B \rightarrow X$ be maps between sets. Their fiber-product $A \otimes_{X} B$ is the collection of points $(a, b)$ in $A \times B$ such that $a$ and $b$ are mapped to the same point in $X$. In the case when $A \rightarrow X$ and $B \rightarrow X$ are graph morphisms, $A \otimes_{X} B$ has a natural graph structure, whose vertices (resp. edges) are pairs of vertices (resp. edges) in $A, B$ that map to 
the same vertex (resp. edge) in $X$. There is a commutative diagram whose arrows are graph morphisms:

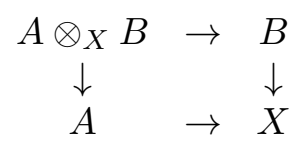

In general $A \otimes_{X} B$ may not be connected even if all of $A, B$ and $X$ are connected (cf. Figure 8). If $B \rightarrow X$ is a covering map of degree $n$, then $A \otimes_{X} B \rightarrow A$ is also a covering map of the same degree. In this case it is also called the pull-back of the covering. The pull-back behaves in a covariant way, i.e. for two consequtive coverings, the pull-back of the composition is canonically homeomorphic to the pull-back by the second map of the pull-back by by first map (see e.g. [27, page 49]).

Note that there is a canonical embedding from $A$ to $A \otimes_{X} A$, whose image is called the diagonal component of $A \otimes_{X} A$. Other components of $A \otimes_{X} A$ (if they exist) are called non-diagonal.

The following lemma is standard, we include a proof for the convenience of the reader.

Lemma 4.2. Suppose $h_{A}: A \rightarrow X$ and $h_{B}: B \rightarrow X$ are immersions. Choose base points $a \in A, b \in B$ such that they are mapped to the same base point $x \in X$.

(1) Let $Z$ be the connected component of $A \otimes_{X} B$ that contains $z=$ $(a, b) \in A \otimes_{X} B$. Then

$$
\pi_{1}(Z, z)=\pi_{1}(A, a) \cap \pi_{1}(B, b)
$$

(we view $\pi_{1}(Z, z), \pi_{1}(A, a)$ and $\pi_{1}(B, b)$ as subgroups of $\pi_{1}(X, x)$.)

(2) for any $g \in \pi_{1}(X, x)$ such that

$$
\pi_{1}(A, a) \cap g \pi_{1}(B, b) g^{-1} \neq\{1\}
$$

there is a component $C$ of $A \otimes_{X} B$ such that

$$
\pi_{1}(C)=\pi_{1}(A, a) \cap g \pi_{1}(B, b) g^{-1}
$$

up to choices of base points.

Proof. The commutativity of the diagram implies $\pi_{1}(Z, z) \subseteq \pi_{1}(A, a) \cap$ $\pi_{1}(B, b)$. To see the other inclusion, let $g \in \pi_{1}(A, a) \cap \pi_{1}(B, b)$ and let $h: \omega \rightarrow X$ be a graph morphism from a (possibly subdivided) circle to $X$ representing the shortest edge loop in $X$ based at $x$ corresponding to $g$. We claim $h$ lifts to an edge loop based at $a$. To see the claim, let

$$
(\tilde{X}, \tilde{x}) \rightarrow(X, x)
$$

be the universal cover of $X$ with a lift $\tilde{x}$ of $x$. Let $\tilde{h}_{A}: \widetilde{A} \rightarrow \widetilde{X}$ be a base pointed lift of $h_{A}$. Since $\widetilde{X}$ and $\widetilde{A}$ are simply connected and $\tilde{h}_{A}$ is an immersion, $h_{A}$ is an embedding and we view $\widetilde{A}$ as a subspace of $\widetilde{X}$. Note that $h$ lifts to an shortest edge path $\widetilde{\omega} \subseteq \widetilde{X}$ whose two endpoints are in $\widetilde{A}$. Hence $\widetilde{\omega} \subseteq \widetilde{A}$ as $\widetilde{A}$ is a subtree. Now the claim follows. Similarly, we can 
also lift $h$ to an edge loop in $B$ based at $b$. This defines an edge loop in $A \otimes_{X} B$ based at $z$. Thus (1) follows.

Now we prove (2). We define $\tilde{h}_{B}: \widetilde{B} \rightarrow \widetilde{X}$ in a way similar to the previous paragraph. Note that $\pi_{1}(A, a)$ stabilizes $\widetilde{A}$ and $g \pi_{1}(B, b) g^{-1}$ stabilizes $g \widetilde{B}$. Let $h \in \pi_{1}(A, a) \cap g \pi_{1}(B, b) g^{-1}$ be a non-trivial element. Then $h$ stabilizes a unique line $\ell \subset \widetilde{X}$ and acts on $\ell$ by translation. The uniqueness of $\ell$ implies that $\ell \subset \widetilde{A}$ and $\ell \subset g \widetilde{B}$. Thus $\widetilde{A} \cap g \widetilde{B}$ is non-empty and is stabilized by $\pi_{1}(A, a) \cap g \pi_{1}(B, b) g^{-1}$. Let $v \in \widetilde{A} \cap g \widetilde{B}$ be a vertex. Then $v$ gives rise to a pair of vertices $a^{\prime} \in A$ and $b^{\prime} \in B$ via $\widetilde{A} \rightarrow A$ and $g \widetilde{B} \rightarrow \widetilde{B} \rightarrow B$ such that $a^{\prime}$ and $b^{\prime}$ are mapped to the same vertex in $X$. Let $K$ be the component of $A \otimes_{X} B$ containing $\left(a^{\prime}, b^{\prime}\right)$. Then any edge path of $K$ can be lifted to an edge path inside $\widetilde{A} \cap g \widetilde{B}$. Thus the universal cover of $K$ can be identified with $\widetilde{A} \cap g \widetilde{B}$ and $\pi_{1} K$ can be identified with $\pi_{1}(A, a) \cap g \pi_{1}(B, b) g^{-1}$ up to a change of base points.

The following is an immediate consequence of Lemma 4.2 .

Corollary 4.3. Let $A \rightarrow X$ be an immersion between connected graphs. Then $\pi_{1} A$ is malnormal in $\pi_{1} X$ if and only if each non-diagonal component of $A \otimes_{X} A$ is a tree (i.e. simply-connected)

Note that the statement of the corollary does not depend on choices of base points in $A$ and $X$, so we omit the base points in the statements.

Now, we record another application of the fiber product, which together with Theorem 2.1 give an algorithm to detect whether a finitely generated subgroup of a finitely generated free group is square free or not.

Corollary 4.4. Let $H \leq F_{n}$ be a finitely generated group, $X_{n}$ the wedge of $n$ circles and $f: X \rightarrow X_{n}$ a graph immersion such that $f_{*}\left(\pi_{1}(X)\right)=H$. Then $H$ is square free in $F_{n}$ if and only if for any two different vertices $x_{1}, x_{2} \in X,\left(x_{1}, x_{2}\right)$ and $\left(x_{2}, x_{1}\right)$ are in different connected components of $X \otimes_{X_{n}} X$.

Proof. If $\left(x_{1}, x_{2}\right)$ and $\left(x_{2}, x_{1}\right)$ are connected by an edge path $\omega \subset X \otimes_{X^{\prime}} X$, then $\omega$ maps to a loop in $X_{n}$ which gives a word $w \in F_{n}$ such that $w \notin H$ (since $\omega$ does not map to a loop in $X$ ) and $w^{2} \in H$ (since the word $w$ travels from $x_{1}$ to $x_{2}$, and it also travels from $x_{2}$ to $x_{1}$ ). Conversely, suppose there exists $w \in F_{n}$ such that $w \notin H$ and $w^{2} \in H$. Note that $w$ stabilizes an embedded line $\ell$ in the universal cover $\widetilde{X}_{n}$ of $X_{n}$. Since $w^{2} \in H, w^{2}$ stabilizes an embedded line $\ell^{\prime}$ in a lift $\widetilde{X}$ of $X$ in $\widetilde{X}_{n}$. Then $\ell^{\prime}=\ell$. Pick a vertex $\tilde{x}_{1} \in \ell^{\prime} \subset \widetilde{X}_{n}$ and let $\tilde{x}_{2}=w \tilde{x}_{1} \in \widetilde{X}_{n}$. Let $x_{i}$ be the image of $\tilde{x}_{i}$ under $\widetilde{X} \rightarrow X$. Then $x_{1} \neq x_{2}$ (since $\left.w \notin H\right)$ and $\left(x_{1}, x_{2}\right)$ and $\left(x_{2}, x_{1}\right)$ are in the same component (since $w^{2} \in H$ ).

\section{Connectedness}

Another consequence of Lemma 4.2 which will be useful is the following. 
Corollary 5.1. Suppose $p: B \rightarrow X$ is a covering map of degree $d>1$ and $f: A \rightarrow X$ is an immersion. If $f$ lifts to an embedding $\tilde{f}: A \rightarrow B$, then the fiber product $A \otimes_{X} B$ is disconnected.

Proof. Note that by the definition of the fiber product, $A$ is contained the fiber product (identifying $A$ with its image under the embedding $\tilde{f}$ ). On the other hand, the fiber product is a cover of $A$, so $A$ itself has to be a connected component of the fiber product, which shows that the fiber product is disconnected when $d>1$.

On the other hand, the following lemma provides a useful condition for when the fiber product is connected.

Lemma 5.2. Let $p$ be a prime and let $f: A \rightarrow X$ be an immersion between two connected graphs such that

$$
f_{X}: H_{1}(A, \mathbb{Z} / p \mathbb{Z}) \rightarrow H_{1}(X, \mathbb{Z} / p \mathbb{Z})
$$

is an isomorphism. Let $X^{\prime} \rightarrow X$ be a regular cover of degree $p$. Then $A \otimes_{X} X^{\prime}$ is connected, $A \otimes_{X} X^{\prime} \rightarrow A$ is a regular cover of degree $p$, and $H_{1}\left(A \otimes_{X} X^{\prime}, \mathbb{Z} / p \mathbb{Z}\right)$ and $H_{1}\left(X^{\prime}, \mathbb{Z} / p \mathbb{Z}\right)$ have the same rank.

Proof. Let $G=\pi_{1}(X, x)$ and let $x^{\prime} \in X^{\prime}$ be a lift of $x$. Then $G^{\prime}=\pi_{1}\left(X^{\prime}, x^{\prime}\right)$ can be identified as the kernel of a surjective homomorphism $h: G \rightarrow \mathbb{Z} / p \mathbb{Z}$. Let $H=\pi_{1}(A, a)$ where $f(a)=x$. Then we have the following commutative diagram:

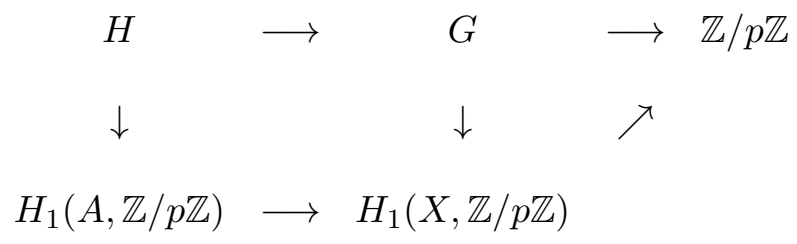

Note that the map $G \rightarrow H_{1}(A, \mathbb{Z} / p \mathbb{Z})$ factors as $G \rightarrow H_{1}(A, \mathbb{Z}) \rightarrow$ $H_{1}(A, \mathbb{Z} / p \mathbb{Z})$, where the first map is the abelianization map, and the second map is tensoring with $\mathbb{Z} / p \mathbb{Z}$. Since $p$ is prime, $G \rightarrow \mathbb{Z} / p \mathbb{Z}$ factors as the composition of two surjective homomorphisms $G \rightarrow H_{1}(X, \mathbb{Z} / p \mathbb{Z}) \rightarrow \mathbb{Z} / p \mathbb{Z}$. Since $H_{1}(A, \mathbb{Z} / p \mathbb{Z}) \rightarrow H_{1}(X, \mathbb{Z} / p \mathbb{Z})$ is an isomorphism, the composition $H \rightarrow g \rightarrow \mathbb{Z} / p \mathbb{Z}$ is surjective. Thus $H \cap G^{\prime}$ is a normal subgroup of in$\operatorname{dex} p$ in $H$. It follows that the connected component of $A \otimes_{X} X^{\prime}$ containing $(a, x)$ is a $p$-sheet regular cover of $A$, thus $A \otimes_{X} X^{\prime}$ can not have other connected components.

Since $H_{1}(A, \mathbb{Z} / p \mathbb{Z})$ and $H_{1}(X, \mathbb{Z} / p \mathbb{Z})$ are isomorphic, $\pi_{1} A$ and $\pi_{1} X$ have the same rank. By Theorem 2.2 and the previous paragraph, $\pi_{1}\left(A \otimes_{X} X^{\prime}\right)$ and $\pi_{1} X^{\prime}$ have the same rank. Thus the lemma follows. 


\section{Gersten's Lemma}

In this section we prove a version of the Adams lemma [1, proved originally for $\mathbb{Z}$. The statement we need in Lemma 6.2 below appears implicitly in the work of Gersten [7] but we provide a short proof for completeness.

Let $p$ be a prime number. Given a ring $R$ with identity 1 , write $R[t]_{p}$ for $R[t] /\left(1-t^{p}\right)$. Suppose $M$ and $N$ are free $R$-modules and $\hat{M}$ and $\hat{N}$ are free $R[t]_{p}$ modules such that $M$ and $\hat{M}$ as well as $\mathbb{N}$ and $\hat{N}$ have the same rank. Given bases $a_{i}$ and $\hat{a}_{i}$ of $M$ and $\hat{M}$, respectively, and $b_{j}$ and $\hat{b_{j}}$ be bases of $N$ and $\hat{N}$, respectively. Write $\phi_{M}$ for the map induced by $a_{i} \mapsto \hat{a}_{i}$ and $t \mapsto 1$ and $\phi_{N}$ for the map induced by $b_{j} \mapsto \hat{b}_{j}$ and $t \mapsto 1$.

We are going to use the following claim

Claim 6.1. Let $M$ and $N$ be free $(\mathbb{Z} / p \mathbb{Z})$-modules and $\hat{M}$ and $\hat{N}$ be free $(\mathbb{Z} / p \mathbb{Z})[t]_{p}$-modules such that $M$ and $\hat{M}$ as well as $N$ and $\hat{N}$ have the same rank and $\varphi_{M}: M \rightarrow \hat{M}, \varphi_{N}: N \rightarrow \hat{N}$ are as above. Suppose $f: M \rightarrow N$ is an $(\mathbb{Z} / p \mathbb{Z})$-homomorphism and $\hat{f}: \hat{M} \rightarrow \hat{N}$ is an $(\mathbb{Z} / p \mathbb{Z})[t]_{p}$-homomorphism such that the following diagram commutes

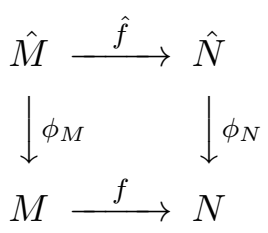

If $f$ is $1-1$, then $\hat{f}$ is 1-1 too.

Proof. Let $\alpha \in \hat{M}$ be a nonzero element. Note that in $\mathbb{Z} / p \mathbb{Z}[t]_{p}$ we have $(1-t)^{p}=1-t^{p}=0$ and choose maximal $k<p$ such that $\alpha=(1-t)^{k} \alpha_{1}$ for some $\alpha_{1}$. Note that $\phi_{M}\left(\alpha_{1}\right) \neq 0$ because otherwise all coordinates of $\alpha_{1}$ would be divisible by $(1-t)$, which contradicts maximality of $k$.

Now, we have $f\left(\phi_{M}\left(\alpha_{1}\right)\right) \neq 0$ because $f$ is 1-1. Thus, $\phi_{N}\left(\hat{f}\left(\alpha_{1}\right)\right) \neq 0$ too. This means that at least one coordinate of $\hat{f}\left(\alpha_{1}\right)$ is not divisible by $(1-t)$. Therefore, at least one coordinate of $(1-t)^{k} \hat{f}\left(\alpha_{1}\right)$ is not divisible by $(1-t)^{p}$ and thus is not zero. Consequently, $\hat{f}(\alpha)=(1-t)^{k} \hat{f}\left(\alpha_{1}\right)$ is nonzero.

Lemma 6.2 (Gersten). Let $X$ and $Y$ be graphs. Let $p$ be a prime and $\pi_{X}: \hat{X} \rightarrow X, \pi_{Y}: \hat{Y} \rightarrow Y$ be $\mathbb{Z} / p \mathbb{Z}$-covers. Suppose $f: X \rightarrow Y$ is a continuous map and $\hat{f}: \hat{X} \rightarrow \hat{Y}$ is a lift

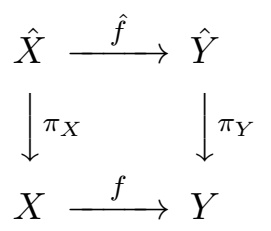

and the map

$$
f_{*}: H_{1}(X, \mathbb{Z} / p \mathbb{Z}) \rightarrow H_{1}(Y, \mathbb{Z} / p \mathbb{Z})
$$


is 1-1. Then the map

$$
\hat{f}_{*}: H_{1}(\hat{X}, \mathbb{Z} / p \mathbb{Z}) \rightarrow H_{1}(\hat{Y}, \mathbb{Z} / p \mathbb{Z})
$$

is 1-1 too.

Proof. Suppose the fundamental group of $X$ isomorphic to $F_{n}$, and the finite cover $\hat{X}$ is corresponding to the kernel of an epimorphism $h: F_{n} \rightarrow \mathbb{Z} / p \mathbb{Z}$. Let $\left\{a_{i}: i \leq n\right\}$ be the generators of $F_{n}$ and let $b_{i}=h\left(a_{i}\right)$. Since $h$ is surjective, at least one of the $b_{i}$, say $b_{1}$ is nontrivial. We assume without loss of generality that $b_{1}=1$. By modifying other $a_{i}$ 's for $i \neq 1$, we can assume that $b_{i}=0$ for $i \neq 1$ (but $a_{i}$ 's still form a basis of $F_{n}$ ).

First note that the following is a set of generators of the kernel of $h$ :

$$
\begin{array}{ccccc}
\left(a_{1}\right)^{p} & & & \\
a_{2}, & a_{1} a_{2}\left(a_{1}\right)^{-1} & \left(a_{1}\right)^{2} a_{2}\left(a_{1}\right)^{-2}, & \ldots & \left(a_{1}\right)^{p-1} a_{2}\left(a_{1}\right)^{1-p} \\
& & \ldots & & \\
a_{n}, & a_{1} a_{n}\left(a_{1}\right)^{-1}, & \left(a_{1}\right)^{2} a_{n}\left(a_{1}\right)^{-2} & \ldots & \left(a_{1}\right)^{p-1} a_{n}\left(a_{1}\right)^{1-p}
\end{array}
$$

This gives a basis for the first homology of $\hat{X}$. Let $\hat{a}_{i}$ be a lift of $a_{i}$. Let $t$ be the action by deck transformation of a generator of $\mathbb{Z} / p \mathbb{Z}$. This gives a structure of a $\mathbb{Z} / p \mathbb{Z}[t]_{p}$-module on the chain group of $\hat{X}$. Write $\hat{M}$ for the $\operatorname{sub}-(\mathbb{Z} / p \mathbb{Z})[t]_{p}$-module generated by $\hat{a}_{i}$ 's.

One checks directly that each element of the above basis of the homology of $\hat{X}$ is contained in $\hat{M}$, thus $H_{1}(\hat{X}, \mathbb{Z} / p \mathbb{Z})$ is contained in $\hat{M}$.

Note that $\hat{M}$ is equal (as a set) to the sub- $(\mathbb{Z} / p \mathbb{Z})$-module generated by $t^{k} \hat{a}_{i}$ where $k$ is ranging between 0 and $p-1$.

Claim 6.3. $\hat{M}$ is a free $(\mathbb{Z} / p) \mathbb{Z}[t]_{p}$-module.

Proof. We show that $\left\{t^{k} \hat{a}_{i}: 0 \leq k \leq p-1,1 \leq i \leq n\right\}$ is linearly independent in the first chain group of $\hat{X}$, viewed as a $\mathbb{Z} / p \mathbb{Z}$-module. It suffices to show that the sub- $(\mathbb{Z} / p \mathbb{Z})$-module $E$ generated by $\left\{t^{k} \hat{a}_{i}\right\}$ has dimension $p n$ (since $p$ is prime, we can think of $\mathbb{Z} / p \mathbb{Z}$ vector spaces rather than $\mathbb{Z} / p \mathbb{Z}$-modules).

Consider the two subspaces of $E$ : $E_{1}$ generated by $\left\{t^{k} \hat{a_{1}}: 0 \leq k \leq p-1\right\}$, and $E_{2}$ generated by $\hat{a_{1}}+t \hat{a_{1}}+\ldots+t^{p-1} \hat{a_{1}}$ and $\left\{t^{k} \hat{a_{i}}: 0 \leq k \leq p-1, i \geq 2\right\}$. Note that $E_{2}$ is equal to the first homology of $\hat{X}$. Hence $\operatorname{dim}\left(E_{2}\right)=1+$ $p(n-1)$ by Theorem 2.2. It is easy to see that $\operatorname{dim}\left(E_{1}\right)=p$. Moreover, $E_{1} \cap E_{2}$ is the line spanned by $\hat{a_{1}}+t \hat{a_{1}}+\ldots+t^{p-1} \hat{a_{1}}$. So

$$
\operatorname{dim}(E)=\operatorname{dim}\left(E_{1}\right)+\operatorname{dim}\left(E_{2}\right)-\operatorname{dim}\left(E_{1} \cap E_{2}\right)=p n .
$$

Now write $M$ for $H_{1}(X, \mathbb{Z} / p \mathbb{Z})$ and let $\hat{M}$ be as above. Note that $M$ is a free $(\mathbb{Z} / p \mathbb{Z})$-module and $\hat{M}$ is a free $(\mathbb{Z} / p \mathbb{Z})[t]_{p}$-module by Claim 6.3 . Let $N$ be the first chain group of $Y$, and let $\hat{N}$ be the first chain group of $\hat{Y}$ (both with coefficients $\mathbb{Z} / p \mathbb{Z}$ ). Note that $N$ is a $(\mathbb{Z} / p \mathbb{Z})$-module and

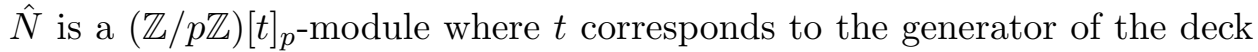
transformation group. $N$ is a free $(\mathbb{Z} / p \mathbb{Z})$-module, by its definition. The 
basis elements of $N$ correspond to the edges of $Y$. The module $\hat{N}$ is free as a $(\mathbb{Z} / p \mathbb{Z})[t]_{p}$-module and if for each edge $e$ of $Y$ we pick a lift $\hat{e}$ of $e$ to $\hat{Y}$, the the set of all $\hat{e}$ forms a basis of $\hat{N}$ as a $(\mathbb{Z} / p \mathbb{Z})[t]_{p}$-module.

Since all spaces considered are graphs, we treat the first homology group as a subgroup of the first chain group. Note that the map $M \rightarrow N$ is injective, since $M$ is first mapped to the first homology group of $Y$ (which is injective by assumption), then the first homology group of $Y$ is inside the first chain group of $Y$.

We have a diagram induced by the continuous maps

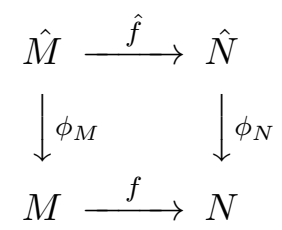

So the map $\hat{f}$ is injective by Claim 6.1 , hence we have injectivity restricted to the homology of $\hat{X}$, which is a subgroup of $\hat{M}$.

Corollary 6.4. Let $p$ be a prime and let $f: A \rightarrow X$ be an immersion between two connected graphs such that

$$
f_{X}: H_{1}(A, \mathbb{Z} / p \mathbb{Z}) \rightarrow H_{1}(X, \mathbb{Z} / p \mathbb{Z})
$$

is an isomorphism. Let $X^{\prime} \rightarrow X$ be a regular cover of degree $p$ and $\hat{f}$ : $A \otimes_{X} X^{\prime} \rightarrow X^{\prime}$ be a lift of $f$.

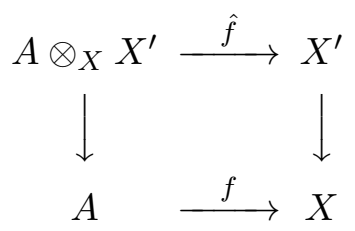

Then $A \otimes_{X} X^{\prime}$ is connected, and

$$
\hat{f}_{*}: H_{1}\left(A \otimes_{X} X^{\prime}, \mathbb{Z} / p \mathbb{Z}\right) \rightarrow H_{1}\left(X^{\prime}, \mathbb{Z} / p \mathbb{Z}\right)
$$

is an isomorphism.

Proof. This follows directly from Lemma 5.2 and Lemma 6.2 since a 1-1 map between $\mathbb{Z} / p \mathbb{Z}$ vector spaces of the same dimension must be an isomorphism.

\section{Extending PARTial AUtomorphisms}

For a natural number $l$, a sequence of $l$-tuples of distinct elements of a set $X$ is a cycle if it is of the form

$$
\left(x_{1}, x_{2}, \ldots, x_{l}\right),\left(x_{2}, \ldots, x_{l}, x_{1}\right), \ldots,\left(x_{l}, x_{1}, \ldots, x_{l-1}\right) .
$$

Given a group $G$ acting on a set $X$, the natural action of $G$ on $X^{l}$ is the coordinatewise action. Note that for $\bar{x}=\left(x_{1}, \ldots, x_{l}\right) \in X^{l}$, the $G$-orbit 
of $\bar{x}$ contains a cycle if and only if there is a $g \in G$ such that $g\left(x_{1}\right)=$ $x_{2}, \ldots, g\left(x_{l}\right)=x_{1}$.

Definition 7.1. Given a set $L$ of natural numbers, an $L$-hypergraph is a relational structure with one relational symbol of arity $l$ for every $l \in L$.

Note that a $\{2\}$-hypergraph is simply a directed graph.

Given a relational symbol $R$ of arity $l$ and a permutation $\sigma \in \operatorname{Sym}(l)$ we write $R_{\sigma}$ for the relation

$$
R_{\sigma}\left(x_{1}, \ldots, x_{l}\right) \quad \text { iff } \quad R\left(x_{\sigma(1)}, \ldots, x_{\sigma(l)}\right) .
$$

Definition 7.2. Suppose $L$ is a set of natural numbers. An $L$-hypergraph $M$ is an $L$-hypertournament if whenever $R$ is an $l$-relational symbol for $l \in L$, then for every tuple of distinct elements $\bar{x} \in M^{l}$ there exists a permutation $\sigma \in \operatorname{Sym}(\{1, \ldots, l\})$ such that

$$
M \models R_{\sigma}(\bar{x})
$$

and for every $R$ and permutation $\sigma$ there does not exists a cycle $\bar{x}_{1}, \ldots, \bar{x}_{l}$ such that that

for every $i \leq l$.

$$
M \models R_{\sigma}\left(\bar{x}_{i}\right)
$$

Note that a $\{2\}$-hypertournament is just a tournament.

The following lemma is a revamp of the analogous equivalence proved by Herwig and Lascar for tournaments [10].

Lemma 7.3. Let $L$ be a nontrivial set of prime numbers. The following are equivalent:

(i) The class of L-hypertournaments has the Hrushovski property.

(ii) Every finitely generated subgroup of $F_{n}$ which is closed under l-roots for all $l \in L$ is closed in the pro- $L^{\perp}$ topology.

Proof. (i) $\Rightarrow$ (ii) Let $H$ be a f.g. subgroup of $F_{n}$ and suppose $H$ is closed under $l$-roots for every $l \in L$. We will show that $H$ is closed in the pro$L^{\perp}$-topology. Let $a \in F_{n}$ be a word which does not belong to $H$. Consider $X=F_{n} / H$ and note that $F_{n}$ acts naturally on $X$. We introduce a structure of an $L$-hypertournament on $X$ as follows.

For every $l \in L$ and a tuple of distinct elements $\bar{x}=\left(x_{1}, \ldots, x_{l}\right) \in X^{l}$, consider the $F_{n}$-orbit of $\bar{x}$ and note that the orbit does not contain a cycle because $H$ is closed under $l$-roots. Indeed, if such a cycle $\bar{x}=\left(w_{1} H, \ldots, w_{l} H\right)$ existed, then for some $g \in F_{n}$ we would have $g^{l} w_{1} H=w_{1} H$, so $w_{1}^{-1} g^{l} w_{1} \in$ $H$, and thus $w_{1}^{-1} g w_{1} \in H$, which would mean that $w_{1} H=w_{2} H$ and contradict the assumption that $\bar{x}$ consists of distinct elements.

This implies that we can choose for each $l \in L$ a collection of orbits which does not contain a cycle and interpret $R_{l}$ as tuples in this collection.

Now choose a subset $X_{0}$ of $X$ which contains the generators of $H$, the word $a$ and all initial subwords of the above. Note that the generators of $F_{n}$ induce partial automorphisms of $X_{0}$. 
By our assumption there exists a finite $L$-hypertournament $Y$ containing $X_{0}$. Then $G=\operatorname{Aut}(Y)$ has no elements of order in $L$ and we get a homomorphism $\varphi$ from $F_{n}$ to $G$ such that all elements of $H$ stabilize $H \in Y$ and $a$ does not stabilize $H$. This implies that $\varphi(a) \notin \varphi(H)$, as needed.

(ii) $\Rightarrow$ (i) Now suppose $M$ is a finite $L$-hypertournament and $P$ is a finite set of partial automorphisms of $M$. Let $k$ be the size of $P$. Let $G_{M}$ be the immersed graph induced by $P$ on $M$. We can assume that $G_{M}$ is connected (extending $P$ if needed). For $x \in M$ let $H_{x}=\pi_{1}\left(G_{M}, x\right)$. Recall that we treat $H_{x}$ as a subgroup of $F_{k}$.

First note that each $H_{x}$ is closed under $l$-roots for every $l \in L$. Indeed, fix $x \in M$ and suppose that $w \in F_{k}$ is such that $w^{l} \in H_{x}$. Let $x_{i}=w^{i}(x)$ with $x_{0}=x$. Since $M$ is an $L$-hypertournament, there is a permutation $\sigma$ such that $M \models\left(R_{l}\right)_{\sigma}\left(x_{0}, \ldots, x_{l-1}\right)$. Then $w$ induces a cycle $\bar{x}_{0}, \ldots, \bar{x}_{l-1}$ starting with $\bar{x}_{0}=\left(x_{0}, \ldots, x_{l-1}\right)$ such that $M \models\left(R_{l}\right)_{\sigma}\left(\bar{x}_{i}\right)$ for all $i<l$, which contradicts the assumption that $M$ is an $L$-hypertournament. Thus, by our assumption, every $H_{x}$ is closed in the pro- $L^{\perp}$ topology.

Choose $x_{0} \in M$. For each $x \in M$ choose a word $w_{x}$ such that $w_{x}\left(x_{0}\right)=x$. For two elements $y, z \in M$ write $w_{z, y}=w_{z} w_{y}^{-1}$.

For each pair of tuples of distinct elements $\bar{y}=\left(y_{0}, \ldots, y_{n-1}\right)$ and $\bar{z}=$ $\left(z_{0}, \ldots, z_{n-1}\right)$ in $M$ such that $M \models R_{n}(\bar{y})$ but $M \not \models R_{n}(\bar{z})$ note that

$$
w_{z_{0}, y_{0}} H_{y_{0}} \cap \ldots \cap w_{z_{n-1}, y_{n-1}} H_{y_{n-1}}=\emptyset .
$$

Since all $H_{y_{i}}$ are closed in the pro- $L^{\perp}$ topology and the latter is compact Hausdorff, there exists a basic open neighborhood of 1, i.e. a normal subgroup $J$ whose index is finite and not divisible by any number in $L$ such that

$$
w_{z_{0}, y_{0}} H_{y_{0}} J \cap \ldots \cap w_{z_{n-1}, y_{n-1}} H_{y_{n-1}} J=\emptyset .
$$

for all tuples $\bar{y}$ and $\bar{z}$ as above and $w_{x}^{-1} w_{y} \notin H_{x_{0}} J$ for any $x \neq y$.

Now consider the subgroup $K=H_{x_{0}} J$ and let $N$ be the set $F_{k} / K$. Since for each $x \in M$ we chose a word $w_{x}$ such that $w_{x}\left(x_{0}\right)=x$, we can map $x \mapsto w_{x} K$ and since $K$ does not contain any $w_{x}^{-1} w_{y}$ for $x \neq y$, this is an embedding. We need to introduce a structure of an $L$-hypertournament on $N$ such that $M$ is a substructure.

We have the natural action of $F_{k}$ on $F_{k} / K$ and write $j: F_{k} \rightarrow \operatorname{Sym}(N)$. Note that the kernel of $j$ contains $J$, so the index of the kernel of $j$ is not divisible by any number in $L$. Write $G=F_{k} / \operatorname{ker}(j)$ and note that $G$ is a finite group without elements of order divisible by a number in $L$, thus its rank is only divisible by numbers in $L^{\perp}$.

Now we can extend the structure from $M$ to $N$ as follows. First, for every tuple $\bar{y}=\left(y_{0}, \ldots, y_{n-1}\right)$ in $M$ such that $M \models R\left(y_{0}, \ldots, y_{n-1}\right)$ extend $R$ to all tuples in the $G$-orbit of $\bar{y}$. Note that $(*)$ implies that this does not change the structure on $M$. Indeed, otherwise there is a $\bar{z}=\left(z_{0}, \ldots, z_{n-1}\right)$ such that $M \models \neg R\left(z_{0}, \ldots, z_{n-1}\right)$. Suppose $g \in F_{k}$ is such that $g$ maps $w_{y_{i}} K$ to $w_{z_{i}} K$, for all $i<n$. Then $g w_{y_{i}} K=w_{z_{i}} K$ for all $i<n$. Since $K=H_{x_{0}} J$ 
we have $w_{y_{i}}^{\prime} \in w_{y_{i}} H_{x_{0}}$ and $w_{z_{i}}^{\prime} \in w_{z_{i}} H_{x_{0}}$ such that

$$
g \in w_{z_{i}}^{\prime} J\left(w_{y_{i}}^{\prime}\right)^{-1}=w_{z_{i}}^{\prime}\left(w_{y_{i}}^{\prime}\right)^{-1} J
$$

since $J$ is normal. Now $w_{z_{i}}^{\prime}\left(w_{y_{i}}^{\prime}\right)^{-1} \in w_{z_{i}, y_{i}} H_{y_{i}}$, so we get a contradiction with (*).

Moreover, since $G$ does not have elements of order divisible by a number in $L$, this does not introduce cycles of order in $L$. Next, to get a hypertournament, we extend the structure to all remaining tuples in $N$ in the same way, i.e. if $\bar{x}=\left(x_{0}, \ldots, x_{n-1}\right)$ is a tuple such that $R_{n}$ does not hold on any permutation of $\bar{x}$, then we extend $R_{n}$ to the $G$-orbit of $\bar{x}$. Again, since $G$ does not contain elements of order in $L$, this defines a hypertournament.

Definition 7.4. Suppose that $G$ is an immersed graph. We say that $G$ is a subtadpole if $G$ has at most one vertex of degree 3 at all other vertices have degree at most 2 .

Note that a connected subtadpole graph looks like a tadpole or a cycle or a tripod or a line. If a subtadpole graph is disconnected, then at most one of its connected components is a tadpole or a tripod and the remaining ones are cycles or lines.

Given a family $P$ of $n$ partial bijections of a set $X$, we say that $P$ forms a subtadpole if the corresponding immersed graph is a subtadpole.

The following proposition is written in the spirit of the previous lemma and gives equivalent conditions to the fact that (ii) in the lemma holds only for cyclic groups.

Proposition 7.5. Let $L$ be a nontrivial set of prime numbers. The following are equivalent:

(i) The class of finite L-hypertournaments has the Hrushovski property for families of partial isomorphisms which form subtadpoles.

(ii) Every cyclic subgroup of $F_{n}$ which is closed under $l$-roots for all $l \in L$ is closed in the pro- $L^{\perp}$ topology.

Proof. (i) $\Rightarrow$ (ii) Let $C$ be a cyclic subgroup of $F_{n}$ and $c \in C$ be its generator. Assume that $C$ is closed under $l$-roots for all $l \in L$. To show that $C$ is closed in the pro- $L^{\perp}$ topology, choose $a \notin C$. We may assume that $a$ does not contain $c^{i}$ as an initial subword for any $i>0$, for otherwise if $a=a^{\prime} c^{i}$, then we can replace $a$ with $a^{\prime}$ (note that we are reading words from right to left as we are talking about left actions).

Consider first the set $X_{1}=F_{n} / C$ and as in the previous proposition introduce a structure of an $F_{n}$-invariant $L$-hypertournament on $X_{1}$, using the assumption that $C$ is closed under $l$-roots for all $l \in L$. Let $k$ be the length of $a$ and let $X=X_{1} \cup \ldots \cup X_{k}$ be a union of $k$ disjoint copies of $X_{1}$. Let $b$ be the longest common initial subword of $c$ and $a$ and let $a=a^{\prime} b$. By our assumption on $a$, we have that $b$ does not contain $c$ as an initial subword. Let $x_{0}=b C$. For each $i<k$ let $x_{i}$ be the copy of $x_{0}$ in $X_{i}$. 
We define a family of partial bijections of $X$. First, if $c_{i}$ is the $i$-th initial subword of $c$, then let the partial bijection corresponding to the $i$-th letter of $c$ map $c_{i-1} C$ to $c_{i} C$. Next, for each $i<k$ if the $i$-th letter of $a^{\prime}$ is $a_{i}$, then let $a_{i}$ map $x_{i}$ to $x_{i+1}$. Note that these partial bijections form a subtadpole on $X$. Now we define a structure of an $L$-hypertournament on $X$ so that all these partial bijections are partial isomorphisms.

Note that the partial action of $F_{n}$ on $X$ induces also a partial action on its $l$-element subsets for each $l \in L$. In order for the partial maps to be partial isomorphisms, we need that the $L$-hypergraph structure is invariant under this partial action. Note that if an orbit of an $l$-tuple is entirely contained in $X_{1}$, then by our construction of the structure on $X_{1}$, the hypergraph relations are invariant on such orbits. If an orbit is not entirely contained in $X_{1}$ but did contain a cycle, then the cycle would have to be contained in $X_{1}$ (by the subtadpole structure), which is impossible. Thus, we can carry over the relations from $X_{1}$ to such orbits without creating cycles. Finally, all other orbits contain only single subsets, so we can extend the definition of the relations on those orbits arbitrarily not creating cycles.

Finally, find a finite $L$-hypertournament $X^{\prime}$ of $X$ which is closed under all subwords of $c$ and under $a$. By our assumption $X^{\prime}$ can be extended to a finite $L$-hypertournament $X^{\prime \prime}$ such that all our partial isomorphisms extend to automorhpisms of $X^{\prime \prime}$. This defines a homomorphisms of $F_{n}$ to the automorphism group of $X^{\prime \prime}$. Since $X^{\prime \prime}$ is an $L$-hypertournament, its automorphism group does not contain elements of order $l$ for any $l \in L$ and thus its rank is divisible only by numbers in $L^{\perp}$. To end the proof, observe that all elements of $C$ stabilize $x_{0}$, while $a$ does not, so this homomorphism does not map $a$ to the image of $C$.

(ii) $\Rightarrow$ (i) This implication is proved exactly as in the previous proposition with the observation that the fundamental group of a subtadpole is cyclic.

\section{A Counterexample}

In this section we prove Theorem 1.6 and Theorem 1.3 .

Let $f: A \rightarrow X$ be the immersion of graphs indicated in Figure 1, where the immersion map preserves orientation and labeling of edges. Note that the image of $f_{*}: \pi_{1} A \rightarrow \pi_{1} X$ is the subgroup generated by $a b a^{-1} b^{-1} a$ and $b$ inside $F_{2}=\langle a, b\rangle$.

Proof of Theorem 1.6. Write $G=\pi_{1}(A)$ for $A$ as above.

Lemma 8.1. $G$ is malnormal in $F_{2}$.

Proof. By Corollary 4.3, it suffices to show each non-diagonal component of $A \otimes_{X} A$ is a tree.

A direct computation as in Figure 2 implies that this is indeed true (note that $A$ has 5 vertices, $3 a$-edges and $3 b$-edges, hence $A \otimes_{X} A$ has 25 vertices, 

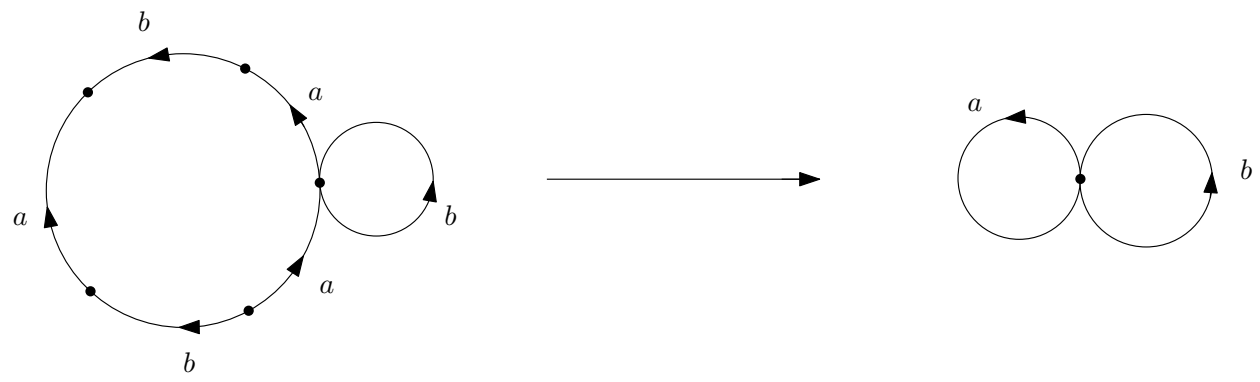

Figure 1. The immersion $f: A \rightarrow X$.
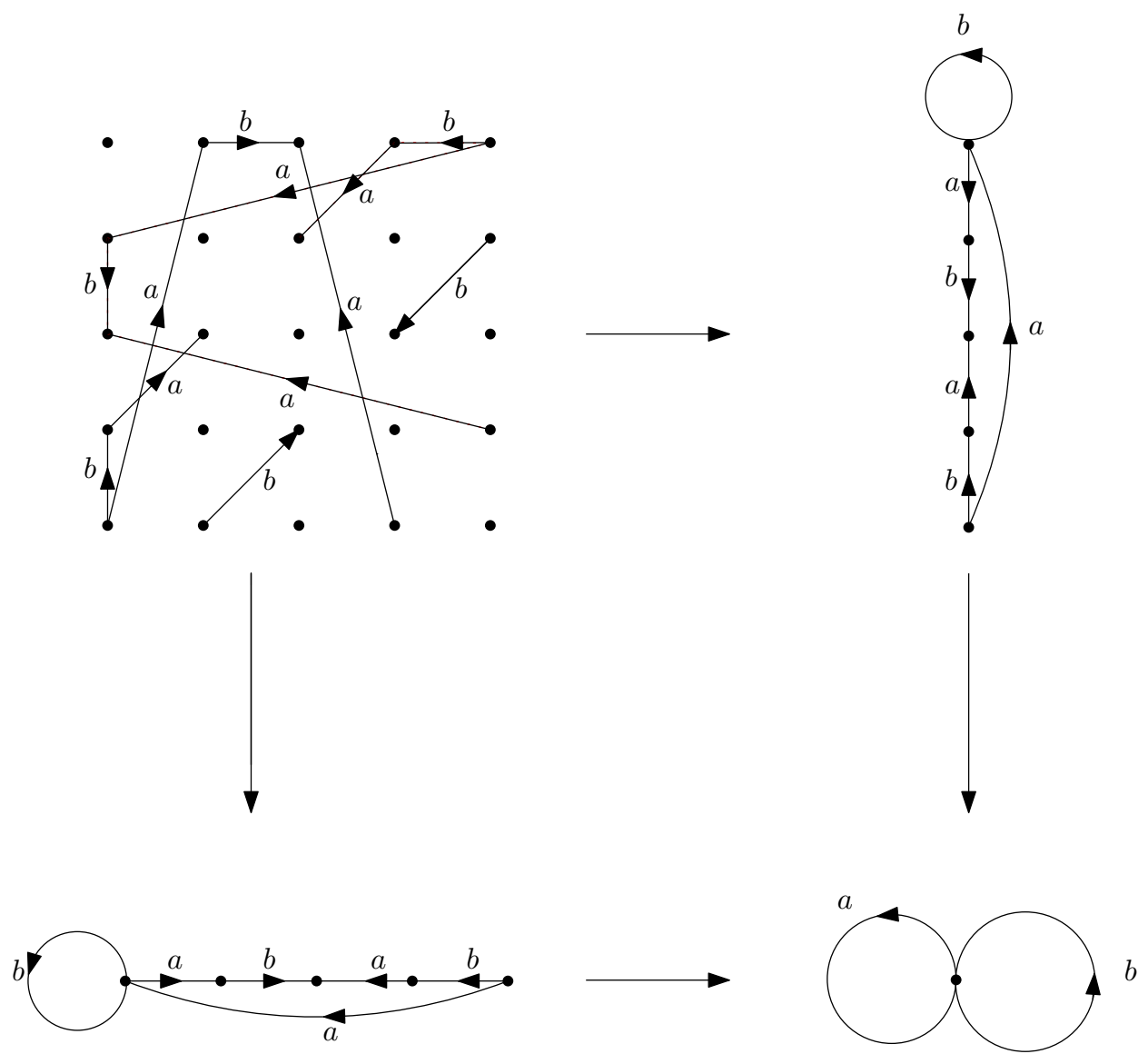

FiguRE 2. Only non-diagonal components of the fiber product are drawn.

$9 a$-edges and $9 b$-edges, moreover, only $6 a$-edges and $6 b$-edges of $A \otimes_{X} A$ are non-diagonal and they are drawn in Figure 2). 
Now we show that $G$ is not closed in any pro- $p$ topology. Fix $p$ and suppose $G$ is closed in the pro- $p$ topology. Since $G$ is malnormal, it is closed under $q$-th roots for all prime $q \neq p$, by Claim 2.5, and we can introduce a structure of an $\{p\}^{\perp}$-hypertnornament on $A$. We do this the same way as in the proof of Lemma 7.3 . since $A$ has 5 vertices, we need to define relations of arity $q=2,3,4,5$. For each such $q$ and each $q$-tuple in $A$ choose one permutation of the tuple to include into the structure. Next, pass through the elements of $G$ to extend the structure so that $G$ acts by partial isomorphisms. The fact that $G$ is closed under $q$-th roots implies that the structure is an $\{p\}^{\perp}$-hypertournament. Abusing notation a bit, let us refer to this hypertournament also by $A$.

If $G$ is closed in the pro- $p$ topology, then by Lemma 7.3 (ii) $\Rightarrow$ (i) (this implication uses only the assumption that the groups $H_{x}$ are closed in the pro- $p$ topology and they are all conjugates of $G$ ), there is a finite $\{p\}^{\perp_{\text {- }}}$ hypertournament $A^{\prime}$ extending the one on $A$ such that both partial maps on $A$ (corresponding to $a$ and $b$ ) extend to isomorphisms of $A^{\prime}$. This makes $A^{\prime}$ a covering of $X$ (the wedge of two circles). Write $\pi_{A^{\prime}}: A^{\prime} \rightarrow X$ for the covering map. Note that $\pi_{1}\left(A^{\prime}\right)$ is closed under $q$-th roots for all prime $q \neq p$ since $A^{\prime}$ is a $\{p\}^{\perp}$-hypertournament.

Now, there is a further finite cover $\pi_{B}: B \rightarrow A^{\prime}$ such that $B$ is a regular connected cover of $X$ (i.e. $B$ corresponds to the biggest normal subgroup of $F_{2}$ contained in the fundamental group of $A^{\prime}$ ). Note since $A^{\prime}$ is nontrivial, $B$ has degree bigger than 1 . Write $H$ for $\pi_{1}(B)$ and note that $H$ is the intersection of finitely many conjugates of $\pi_{1}\left(A^{\prime}\right)$, hence it is closed under $q$-th roots for all prime $q \neq p$. As $H$ is normal in $F_{2}$, the quotient $F_{2} / H$ is a $p$-group and there exists a subnormal series

$$
F_{2} \triangleright \ldots H_{1} \triangleright H_{0}=H
$$

such that each $H_{i+1} / H_{i} \simeq \mathbb{Z} / p \mathbb{Z}$. This corresponds to a sequence of intermediate subcovers

$$
X=X_{0} \leftarrow \ldots \leftarrow B
$$

such that each $X_{i+1} \rightarrow X_{i}$ is a regular $\mathbb{Z} / p \mathbb{Z}$ cover.

Now, note that $A$ is connected and the map

$$
f_{*}: H_{1}(A, \mathbb{Z} / p \mathbb{Z}) \rightarrow H_{1}(X, \mathbb{Z} / p \mathbb{Z})
$$

ils an isomorphism because the generators of the first homology of $A$ are mapped to the generators of the first homology of $X$. Inductively using Lemma 5.2 and Corollary 6.4, we see that each $A \otimes_{X} X_{i}$ is connected and the map from $H_{1}\left(A \otimes_{X} X_{i}, \mathbb{Z} / p \mathbb{Z}\right)$ to $H_{1}\left(X_{i}, \mathbb{Z} / p \mathbb{Z}\right)$ is an isomorphism. In particular, $A \otimes_{X} B$ is connected.

On the other hand, note that since $A^{\prime}$ extends $A$, the map $f: A \rightarrow X$ lifts to an embedding $f^{\prime}: A \rightarrow A^{\prime}$. Thus, by Corollary 5.1 $A \otimes_{X} A^{\prime}$ is disconnected. Thus, $\left(A \otimes_{X} A^{\prime}\right) \otimes_{A^{\prime}} B$ is disconnected as well. But, again, by covariance of the pull-back, the latter is homeomorphic to $A \otimes_{X} B$. This contradicts the previous paragraph and ends the proof. 


\section{Cyclic subgroups}

Finally, in this section we prove Theorem 1.5 and Theorem 1.2 .

Note that every cyclic subgroup of $F_{n}$ is contained in a maximal cyclic group. Indeed, if $C<F_{n}$ is cyclic and generated by $c$, then a maximal cyclic subgroup of $F_{n}$ containing $C$ can be found by finding $d \in F_{n}$ of minimal length such that $d^{k}=c$ for some positive integer $k$.

Lemma 9.1. If $A \subset F_{n}$ is a maximal cyclic subgroup, then $A$ is closed in the pro-p topology for any prime $p$.

Proof. Suppose $A=\langle a\rangle$. Then the maximality of $A$ implies that $A$ is the centralizer of $a$ in $F_{n}$. Pick $x \notin A$ and let $g=[x, a]$. By the fact $F_{n}$ is residually $p$, there exists a finite $p$-group $F$ and a homomorphism $\phi: F_{n} \rightarrow F$ such that $\phi(g)$ is non-trivial. Thus $\phi(x)$ does not commute with $\phi(a)$. Since $\phi(A)$ is a cyclic subgroup generated by $\phi(a)$, we get that $\phi(x) \notin \phi(A)$.

Now we prove Theorem 1.5 .

Proof of Theorem 1.5. Let $A$ be a maximal cyclic subgroup containing $C$. Suppose $i=[A: C]$ and let $g \notin C$. We will separate $g$ from $C$.

Case 1: If $g \notin A$, by Lemma 9.1, we can find a $p$-group $F$ for $p \in L^{\perp}$ and a homomorphism $\phi: G \rightarrow F$ such that $\phi(g) \notin \phi(A)$, hence $\phi(g) \notin \phi(C)$.

Case 2: If $g \in A \backslash C$, we let $A=\langle a\rangle$ and $C=\left\langle a^{i}\right\rangle$. Then $g=a^{i j+m}$ for $j \in \mathbb{Z}$ and $1 \leq m<i$. Let $p$ be a prime factor of $i$. We claim $p \in L^{\perp}$. Indeed, otherwise there is $l \in L$ such that $p \mid l$. Suppose $l=p r_{1}$ and $i=p r_{2}$. Let $q=\langle i, l\rangle$ be the least common multiple of $i$ and $l$. Suppose $q=l r$. Now we consider the element $a^{r}$. It is clear that $\left(a^{r}\right)^{l}=a^{q} \in C$. However,

$$
r=\frac{q}{l}=\frac{\langle i, l\rangle}{l}=\frac{p\left\langle r_{1}, r_{2}\right\rangle}{p r_{1}}=\frac{\left\langle r_{1}, r_{2}\right\rangle}{r_{1}} \leq r_{2}<i .
$$

Thus $a^{r} \notin C$. This contradicts that $C$ is closed under $l$-roots.

Since $F_{n}$ is residually $p$, there is a finite $p$-group $F$ and a homomorphism $\phi: G \rightarrow F$ such that $\bar{a}=\phi(a)$ is non-trivial. We claim $\phi(g) \notin \phi(C)$. Indeed, otherwise there is an integer $s$ such that $\phi(g)=\bar{a}^{i j+m}=\bar{a}^{i s}$. Hence $\bar{a}^{i(j-s)+m}$ is trivial. Since $\bar{a}$ is a non-trivial element in a $p$-group, it has order equal to a power of $p$. In particular $p \mid i(j-s)+m$. Since $p \mid i$ by construction and $p \nmid m$, we reach a contradiction. Thus $\phi(g) \notin \phi(C)$ and we are done.

Proof of Theorem 1.2. Note that if $\varphi_{1}, \ldots, \varphi_{n}$ have pairiwse disjoint domains and pairwise disjoint ranges, then every vertex in the immersed graph induced by them has degree at most 2 and in particular is a subtadpole (in fact, it is a union of circles and lines). Thus, the corollary follows from Theorem 1.5 and Proposition 7.5 .

The case $L=\{2\}$ in the above corollary corresponds to the case considered by Herwig and Lascar in [10]. 


\section{REFERENCES}

[1] J. F. Adams. A new proof of a theorem of W. H. Cockcroft. J. London Math. Soc., 30:482-488, 1955.

[2] Gregory L. Cherlin. The classification of countable homogeneous directed graphs and countable homogeneous n-tournaments. Mem. Amer. Math. Soc., 131(621), 1998.

[3] Siniora D. Automorphism groups of homogeneous structures. PhD thesis, University of Leeds.

[4] Siniora D. and S. Solecki. Coherent extension of partial automorphisms, free amalgamation, and automorphism groups. preprint, arXiv:1705.01888.

[5] Hubiķa J. Konečný M. Evans, D. and J. Nešetřil. EPPA for two-graphs. 2018. draft.

[6] Hubička J. Evans, David M. and J. Nešetřil. Ramsey properties and extending partial automorphisms for classes of finite structures. preprint.

[7] S. M. Gersten. Conservative groups, indicability, and a conjecture of Howie. J. Pure Appl. Algebra, 29(1):59-74, 1983.

[8] Marshall Hall, Jr. Coset representations in free groups. Trans. Amer. Math. Soc., 67:421-432, 1949.

[9] Hatcher. Algebraic Topology. Cambridge University Press, 2002.

[10] Bernhard Herwig and Daniel Lascar. Extending partial automorphisms and the profinite topology on free groups. Trans. Amer. Math. Soc., 352(5):1985-2021, 2000.

[11] Ian Hodkinson and Martin Otto. Finite conformal hypergraph covers and Gaifman cliques in finite structures. Bull. Symbolic Logic, 9(3):387-405, 2003.

[12] Ehud Hrushovski. Extending partial isomorphisms of graphs. Combinatorica, 12(4):411-416, 1992.

[13] Konečny M. Hubička J. and J. Nešetřil. A combinatorial proof of the extension property for partial isometries. preprint.

[14] May J.P. A concise course in algebraic topology. Chicago Lectures in Mathematics. The University of Chicago Press, Chicago, 1999.

[15] Alexander S. Kechris and Christian Rosendal. Turbulence, amalgamation, and generic automorphisms of homogeneous structures. Proc. Lond. Math. Soc. (3), 94(2):302350, 2007.

[16] Thomas Koberda. Right-angled Artin groups and their subgroups. notes available at http://faculty.virginia.edu/Koberda/raagcourse.pdf.

[17] A. H. Lachlan and Robert E. Woodrow. Countable ultrahomogeneous undirected graphs. Trans. Amer. Math. Soc., 262(1):51-94, 1980.

[18] D. Lascar. Automorphism groups of saturated structures; a review. In Proceedings of the International Congress of Mathematicians, Vol. II (Beijing, 2002), pages 25-33. Higher Ed. Press, Beijing, 2002.

[19] Doucha M. and Malicki M. Generic representations of countable groups. preprint, arXiv: 1710.08170 .

[20] Pawliuk M. and Sokic M. Amenability and unique ergodicity of automorphism groups of countable homogeneous directed graphs. Ergodic Theory and Dynamical Systems. to appear.

[21] Lionel Nguyen Van Thé. A survey on structural Ramsey theory and topological dynamics with the Kechris-Pestov-Todorcevic correspondence in mind. Zb. Rad. (Beogr.), 17(25)(Selected topics in combinatorial analysis):189-207, 2015.

[22] Vladimir G. Pestov. A theorem of Hrushovski-Solecki-Vershik applied to uniform and coarse embeddings of the Urysohn metric space. Topology Appl., 155(14):1561-1575, 2008.

[23] Luis Ribes and Pavel Zalesskii. Profinite groups, volume 40 of Ergebnisse der Mathematik und ihrer Grenzgebiete. 3. Folge. A Series of Modern Surveys in Mathematics [Results in Mathematics and Related Areas. 3rd Series. A Series of Modern Surveys in Mathematics]. Springer-Verlag, Berlin, second edition, 2010. 
[24] Christian Rosendal. Finitely approximable groups and actions part I: The RibesZalesskiupproperty. J. Symbolic Logic, 76(4):1297-1306, 2011.

[25] Marcin Sabok. Automatic continuity for isometry groups. arXiv:1312.5141.

[26] Sławomir Solecki. Extending partial isometries. Israel J. Math., 150:315-331, 2005.

[27] Norman Steenrod. The topology of fibre bundles. Princeton Landmarks in Mathematics. Princeton University Press, Princeton, NJ, 1999. Reprint of the 1957 edition, Princeton Paperbacks.

[28] A. M. Vershik. Globalization of the partial isometries of metric spaces and local approximation of the group of isometries of Urysohn space. Topology Appl., 155(14):1618-1626, 2008.

Max Planck Institute for Mathematics Vivatsgasse 7, 53111 Bonn, Germany

E-mail address: jingyin@mplm-bonn.mpg.de

Department of Mathematics and Statistics, University of Calgary, 612 Campus Place N.W., 2500 University Drive NW Calgary, Alberta, Canada $\mathrm{T} 2 \mathrm{~N} 1 \mathrm{~N} 4$

E-mail address: mpawliuk@ucalgary.ca

Department of Mathematics and Statistics, McGill University, 805, SherBrooke Street West Montreal, Quebec, Canada H3A 2 K6

Institute of Mathematics, Polish Academy of Sciences, Śniadeckich 8, 00655 WarszaWa, Poland

E-mail address: marcin.sabok@mcgill.ca

Department of Mathematics and Statistics, McGill University, 805, SherBrooke Street West Montreal, Quebec, Canada H3A 2K6

E-mail address: daniel.wise@mcgill.ca 